Boise State University

ScholarWorks

Mechanical and Biomedical Engineering Faculty

Publications and Presentations

Department of Mechanical and Biomedical

Engineering

$4-2020$

\title{
Computational Approach to Correcting Joint Instability in Patients with Recurrent Patellar Dislocation
}

\author{
Oliver Alvarez \\ Boise State University \\ Robert N. Steensen \\ Mount Carmel Health System \\ Paul J. Rullkoetter \\ University of Denver \\ Clare K. Fitzpatrick \\ Boise State University
}

This is the peer reviewed version of the following article:

Alvarez, O., Steensen, R.N., Rullkoetter, P.J. \& Fitzpatrick, C.K. (2020). Computational Approach to Correcting Joint Instability in Patients with Recurrent Patellar Dislocation. Journal of Orthopaedic Research, 38(4), 768-776.

which has been published in final form by Wiley Periodicals, Inc. on behalf of the Orthopaedic Research Society at https://doi.org/10.1002/jor.24526. This article may be used for non-commercial purposes in accordance with Wiley Terms and Conditions for Self-Archiving. 


\title{
A Computational Approach to Correcting Joint Instability in Patients with Recurrent Patellar Dislocation
}

\author{
Oliver Alvarez \\ Mechanical and Biomedical Engineering \\ Boise State University \\ Boise, ID, USA \\ Paul J. Rullkoetter \\ Center for Orthopaedic Biomechanics \\ University of Denver \\ Denver, CO, USA
}

\author{
Robert N. Steensen \\ Mount Carmel Health System \\ Columbus, $\mathrm{OH}$ \\ Clare K. Fitzpatrick* \\ Mechanical and Biomedical Engineering \\ Boise State University \\ Boise, ID, USA \\ clarefitzpatrick@boisestate.edu
}

\begin{abstract}
All authors have read and approved the final submitted manuscript. Contributions to authorship are as follows: Oliver Alvarez was responsible for running the computational analysis, interpretation of computational results and preparing the initial draft of this manuscript. Robert Steensen was responsible for guiding clinical inputs to the models and classifying the patients for abnormality and contributed to reviewing the manuscript. Paul Rullkoetter contributed to the initial concept of this study, subject-specific model development, and reviewing the manuscript. Clare Fitzpatrick contributed to the initial concept for this study, the design of the computational study, analysis and interpretation of results, and critically revised the initial manuscript draft.
\end{abstract}

\begin{abstract}
Patellar dislocation is a debilitating injury common in active adolescents and young adults. Conservative treatment after initial dislocation is often recommended, but almost half of these patients continue to suffer from recurrent dislocation. The objective of this study is to compare preoperative patellofemoral joint stability with stability after a series of simulated procedures, including restorative surgery to correct to pre-injury state, generic tibial tubercle osteotomy, patient-specific reconstructive surgery to correct anatomic abnormality, less invasive patientspecific surgery and equivalent healthy controls. Three-dimensional, subject-specific finite element models of the patellofemoral joint were developed for 28 patients with recurrent patellar dislocation. A $50 \mathrm{~N}$ lateral load was applied to the patella to assess the lateral stability of the patellofemoral joint at $10^{\circ}$ intervals from $0^{\circ}$ to $40^{\circ}$ flexion. Medial patellofemoral ligament reconstruction, along with reconstructive procedures to correct anatomic abnormality were simulated. Of all the simulations performed, the healthy equivalent control models showed the least patellar internal-external rotation, medial-lateral translation, and medial patellofemoral ligament restraining load during lateral loading tests. Isolated restorative medial patellofemoral ligament reconstruction was the surgery that resulted in the most patellar internal-external rotation, medial-lateral translation, and medial patellofemoral ligament reaction force across all flexion angles. Patient-specific reconstruction to correct anatomic abnormality was the only surgical group to have non-significantly different results compared to the healthy equivalent control group across all joint stability metrics evaluated.
\end{abstract}

Clinical Significance: This study suggests patient-specific reconstructive surgery that corrects underlying anatomic abnormalities best reproduces the joint stability of an equivalent healthy control when compared to pre-injury state, generic tibial tubercle osteotomy, and less invasive patient-specific surgery.

Keywords: patellar dislocation; finite element; patellar instability; patellofemoral joint mechanics; surgery 
This is an author-produced, peer-reviewed version of this article. The final, definitive version of this document can be found online at Journal of Orthopaedic Research, published by Wiley Periodicals, Inc. on behalf of the Orthopaedic Research Society. Copyright restrictions may apply. https://doi.org/10.1002/jor.24526

\section{Introduction}

Lateral patellar dislocation is one of the most common acute knee injuries in young active people and accounts for 3\% of all knee injuries. ${ }^{1}$ Over 20,000 persons per year are affected by an initial incidence of patellar dislocation in the United States. ${ }^{2}$ The typical mechanism of injury occurs with the quadriceps engaged and the femur rotated internally, ${ }^{3}$ with dislocation occurring at $20^{\circ}$ to $30^{\circ}$ of tibiofemoral (TF) flexion. Those suffering from recurring patellar dislocation typically experience persistent symptoms of patellar instability, anterior knee pain, swelling and patellofemoral (PF) osteoarthritis, which can significantly impact patient quality of life. ${ }^{2,4,5}$ Conservative treatment through physical therapy after initial dislocation is often recommended, ${ }^{5,6}$ but results are frequently unsatisfactory. Approximately half of these first-time dislocation patients (reports range from 15-72\%) go on to experience a subsequent dislocation or multiple dislocation events. ${ }^{4,7,8}$ Subsequent dislocation events have potential to further damage the soft tissues or articular cartilage surfaces of the PF joint and non-operative treatment has shown high risk of patellofemoral osteoarthritis in the longer term. ${ }^{4,6}$

The standard protocol for treating these patients after conservative treatment fails is medial patellofemoral ligament (MPFL) reconstruction. The MPFL is commonly ruptured (in up to $94 \%$ of patients) during the initial incidence of patellar dislocation. ${ }^{79}$ However, since there are often predisposing anatomic factors present, there is little consensus on a standard protocol to determine when and which surgeries to perform, especially for patients with multiple abnormal factors. ${ }^{10}$ A targeted patient-specific approach which directly addresses risk factors for patellar dislocation has potential to improve surgical outcomes for patients with recurrent instability. Prior in vivo, in vitro, and computational studies have evaluated factors contributing towards patellar dislocation and investigated potential interventions including sulcus deepening trochleoplasty, medial patellofemoral ligament reconstruction or tibial tubercle osteotomy. ${ }^{1,11-18}$ However, to the author's knowledge, no prior studies have compared patellar stabilizing procedures, including MPFL reconstruction, trochleoplasty, and tibial tubercle osteotomy, across a series of patientspecific computational analyses. This approach allows for direct quantitative comparison of resulting stability across these different surgical procedures.

Factors thought to contribute to recurrent patellar instability are wide-ranging. Prior clinical studies have examined four factors (patellar height, trochlear morphology, tibial tubercle-trochlear groove (TT-TG) distance and quadriceps angle) and their prevalence and combined prevalence of abnormal anatomic factors. ${ }^{19}$ Multifactorial data from a group of 60 patients with recurrent lateral dislocation and 120 controls, showed that almost $60 \%$ of the patient group had two or more abnormal factors, compared to $1.6 \%$ of the control group. ${ }^{19}$ Studies report trochlear dysplasia, patella alta and greater TT-TG distance in patients with patellar instability as compared to healthy control subjects. ${ }^{19,20}$ Despite these factors commonly cited in the literature, patients may undergo multiple surgical procedures that fail to treat these underlying anatomic abnormalities. ${ }^{8,21,22}$ Patients with recurrent dislocation typically have abnormalities of knee anatomy and soft tissue integrity, with substantial inter-subject variability. Clinical interventions that are tailored to the anatomy and mechanics of the individual patient may have benefit in optimizing post-operative joint stability. ${ }^{23-25}$

Patients with persistent symptoms of patellar instability are candidates for surgical interventions, with treatment options that include MPFL reconstruction, tibial tubercle osteotomy, sulcus-deepening trochleoplasty, or combinations of these procedures. ${ }^{1,26-28}$ However, currently, there is no universally accepted algorithm for choosing the appropriate procedure(s) for a particular individual. ${ }^{10,29-31}$ Studies that evaluate MPFL reconstruction (MPFLR) in patients without identifying predisposing factors make it difficult to extrapolate their success to patients with one or more factors. ${ }^{32,33}$ Little research has been done to determine the minimal invasive procedure to prevent recurrent patellar dislocation on symptomatic knees. Researchers have compared the outcomes of different surgeries, ${ }^{30,25,34}$ however, the patient-specific indicators for which surgery to perform is relatively unknown. ${ }^{29}$ Furthermore, studies often use subjective measures to compare pre- and post-operative performance, ${ }^{8}$ with patients often unable to return to preinjury performance despite reducing further patellar dislocation. ${ }^{27}$

Although clinical studies provide vital in vivo information about joint anatomy, mechanics, and the efficacy of treatment plans, there is a large amount of inter-subject variability that makes it difficult to use clinical data to determine treatment for an individual. Computational models can provide an ideal complement to clinical data..$^{35,36}$ Computational simulation can be used to perform virtual surgery ${ }^{11-14,26,37}$ to determine optimal treatment on a patientspecific basis - alternative mutually exclusive procedures can be simulated on the same patient. Joint biomechanics can be evaluated and compared between surgical simulations and preoperative conditions under uniform loading conditions. The objective of this study is to compare preoperative patellofemoral (PF) joint stability with stability after restorative surgery to correct to pre-injury state, generic tibial tubercle osteotomy, patient-specific 
This is an author-produced, peer-reviewed version of this article. The final, definitive version of this document can be found online at Journal of Orthopaedic Research, published by Wiley Periodicals, Inc. on behalf of the Orthopaedic Research Society. Copyright restrictions may apply. https://doi.org/10.1002/jor.24526

reconstructive surgery to correct anatomic abnormality, less invasive patient-specific surgery and equivalent healthy controls. This study will serve as an aid to orthopedic surgeons in determining the optimal subject-specific treatment to stabilize the PF joint in patients with recurrent patellar dislocation.

\section{Materials and Methods}

Three anatomic criteria, which have been found to differ significantly between patients with recurrent lateral patellar dislocation and controls were selected for evaluation in the current study. These criteria include trochlear geometry, patellar tendon length and medial-lateral tibial tuberosity position. Trochlear dysplasia, patella alta and laterally elevated tibial tuberosity position are commonly reported in patients with recurrent dislocation. ${ }^{19,20}$ There are a variety of definitions which may be used for trochlear dysplasia, patella alta and tuberosity position, ${ }^{19,37}$ the specific factors used in this study were sulcus angle (trochlear geometry) and Insall-Salvati ratio (patellar tendon length), and TT-TG distance (tibial tuberosity position). These were chosen as factors that are frequently reported in other work and could be easily measured and modified in a computational model. ${ }^{19,20}$

A dynamic three-dimensional finite element (FE) model of a PF joint was developed in Abaqus/Explicit (Simulia, Providence, RI). The model is based on a published FE model of an isolated PF joint ${ }^{35}$ and includes femur, tibia, and patella bones, femoral, tibial and patellar articular cartilage, patellar tendon and quadriceps tendon and muscle. The quadriceps muscles were differentiated into rectus femoris (RF), vastus intermedius (VI), vastus lateralis (VL) and vastus medialis $(\mathrm{VM})$ bundles. ${ }^{35,38}$

Magnetic resonance images of knee anatomy from 28 subjects (mean age \pm standard deviation: $25.5 \pm 10.1$ years) with recurrent patella dislocation were obtained under Institutional Review Board approval from Mount Carmel Health System. Femoral, tibial, and patellar bony surfaces and cartilage were extracted via segmentation using commercial software. Full knee joints were aligned to a local femoral coordinate system using an iterative closest point (ICP) algorithm implemented in MATLAB (Mathworks, Natick, MA). Muscle geometry could not be visualized clearly from the MR images, and so was based on muscle attachment sites and lines of action from published cadaveric studies. ${ }^{38}$ Bones were represented with two-dimensional (2D) rigid triangular shell elements. Patellar, femoral and tibial articular cartilage were modeled as fully deformable isotropic elastic, using eight-noded hexahedral elements (Figure 1). The patellar tendon was modeled as six non-linear springs. Quadriceps tendons were modeled as twodimensional membranes (quadrilateral elements) with embedded fiber-reinforced springs to facilitate wrapping of the tendon around the femoral bone and cartilage in later flexion (Figure 1).

Quasi-static simulations were performed at $10^{\circ}$ intervals from $0^{\circ}$ to $40^{\circ} \mathrm{TF}$ flexion. This flexion range was selected as patellar dislocation typically occurs early in flexion before the patella becomes constrained by the femoral trochlear groove. ${ }^{35} \mathrm{TF}$ alignment at each flexion angle was fully prescribed, while the patella was kinematically unconstrained in all DOFs, with restraint provided by the patellar tendon, femoral geometry and musculature. As the MPFL is ruptured in the majority of cases of patellar dislocation, ${ }^{39,40}$ the MPFL was not included in the pre-operative models. However, the MPFL was reconstructed in each surgical (post-operative) simulation since the MPFL is a key component in patellar stabilization. ${ }^{10}$ In these post-operative simulations, the MPFL was represented with mechanical properties and attachment sites of the native MPFL in order to simulate a procedure that restores the MPFL to a preinjury state.

In each simulation, a $400 \mathrm{~N}$ load was applied to the quadriceps muscles to bring articular surfaces into contact. ${ }^{35}$ The load was distributed with a ratio of 15:20:40:25 across the RF, VI, VL, and VM muscles, respectively. ${ }^{38}$ After the knee was flexed to the prescribed angle, a $50 \mathrm{~N}$ load was applied laterally to the patella to assess the stability of the PF joint. ${ }^{1,15-17}$ From the simulations, PF forces and alignment at each quasi-static position were extracted from the analyses after the $50 \mathrm{~N}$ lateral load application (Figure 2). Specifically, patellar medial-lateral (M-L) alignment, patellar internal-external (I-E) angle, lateral constraint force of the trochlea and, in post-operative models, MPFL load as these are metrics associated with lateral constraint of the PF joint. ${ }^{35}$ Patella M-L alignment and I-E rotation measure mobility of the patella, while MPFL load and trochlear constraint force indicate soft tissue and bony anatomic structure restraint to patellar dislocation, respectively. The trochlear constraint force is the M-L reaction force on the trochlear groove to constrain the patella in the trochlear groove.

Each of the 28 patients had the three factors under investigation measured: patella height, TT-TG distance, sulcus angle. The following abbreviations were used to identify subjects with abnormal factors: P for patella alta, Q for abnormally increased TT-TG distance, and S for trochlear dysplasia. For each subject, abnormal factors were 
This is an author-produced, peer-reviewed version of this article. The final, definitive version of this document can be found online at Journal of Orthopaedic Research, published by Wiley Periodicals, Inc. on behalf of the Orthopaedic Research Society. Copyright restrictions may apply. https://doi.org/10.1002/jor.24526

identified from MR scans by an experienced orthopaedic surgeon (RNS) and were classified as described in a previous publication (Figure 3). ${ }^{19}$ Insall-Salvati ratio was measured on a sagittal MR image through the midline of the patella, with patellar tendon length measured from the distal-most point on the patellar bone to the most prominent point on the tibial tubercle. Bony TT-TG distance was assessed between the most prominent point of the tibial tuberosity and the deepest bony point of the trochlear groove (or apex in the case of convex trochleae), perpendicular to the tangent to the bony borders of the posterior condyles on axial MR scans. ${ }^{19}$ The shape of the proximal trochlea was assessed on the 3 most proximal axial images demonstrating articular cartilage. The cartilaginous contour of the trochlea was qualitatively categorized as concave, flat, or convex. A flat or convex trochlea was considered to represent trochlear dysplasia. ${ }^{19}$ The trochlear groove was also measured on bone surface from a skyline view with the knee flexed at $0^{\circ}$, $30^{\circ}, 60^{\circ}$ and $90^{\circ}$, to allow for smooth sulcus deepening of the patient-specific femur bone and cartilage along the entire trochlear groove.

\section{Virtual Surgical Intervention}

The purpose of virtual surgery was to quantitatively determine the optimal surgery on a subject-specific basis. First, a commonly used restorative procedure, medial patellofemoral ligament reconstruction (MPFLR) was simulated on each subject-specific patient model. In this study, MPFLR was represented with properties representative of the native MPFL to simulate restoration to pre-injury state. Subsequently, a series of tibial tubercle distal transfer (TT-D), tibial tubercle medial transfer (TT-M), and sulcus-deepening trochleoplasty (TP) procedures were simulated (Figure 4). A comparison was then made between pre-operative (no surgery), pre-injury (MPFLR), generic tibial tubercle osteotomy (MPFLR + TT-M), patient-specific reconstruction, less invasive patient-specific surgery, and the equivalent healthy control group. In the equivalent healthy control group, all three anatomic parameters under investigation in this study, regardless of initial values, were restored to values equivalent to the mean values of a healthy control group (InsallSalvati ratio $=1.07$; TT-TG distance $=12 \mathrm{~mm}$; sulcus angle $\left.=138^{\circ}\right) .{ }^{19}$ The purpose of the equivalent healthy control group was to set a patient-specific benchmark for the PF force and alignment metrics of a stable joint that other virtual surgeries may be compared against. In the patient-specific reconstruction, all present subject-specific abnormal factors were restored to values equivalent to the mean values of a healthy control group. Less invasive patient-specific surgery was defined similar to the patient-specific reconstruction, except that the most invasive procedure for that patient was not performed. For example, if the subject-specific reconstruction required MPFLR, tibial tubercle osteotomy, and trochleoplasty, the less invasive procedure would include just MPFLR and tibial tubercle osteotomy. If the subjectspecific reconstruction required MPFLR and tibial tubercle osteotomy, the less invasive procedure involved only MPFLR. The rationale behind simulating the pre-injury, generic tibial tubercle osteotomy and less invasive patientspecific surgery was to determine if a generic or less invasive surgery can provide equivalent stability restoration as the equivalent healthy control group.

At full extension, patellar lateral displacement is predominantly restrained by the soft tissues. ${ }^{38}$ When the patella dislocates laterally, the MPFL, which provides $50-67 \%$ of soft tissue restraint,,${ }^{7,9}$ is damaged or ruptured in the majority of incidences ${ }^{39,40}$ which further increases the possibility of recurrent dislocation. ${ }^{6}$ MPFL reconstruction is considered a core component of most patellar stabilizing procedures. ${ }^{10}$ The MPFL was modeled as a two-dimensional membrane with embedded fiber-reinforced spring to facilitate wrapping of the ligament around the femoral bone, with properties of a healthy intact MPFL. ${ }^{41}$ MPFLR has been performed with semitendinosus, gracilis, quadriceps tendon and synthetic grafts. ${ }^{32}$ Using data from a healthy intact MPFL serves as a baseline MPFL representation since graft material is typically stronger and stiffer than the original MPFL. Native MPFL properties also serve to represent a true "restorative" procedure by restoring the properties of the MPFL to its pre-injury state. The MPFL femoral insertion area covers a triangular space between the adductor tubercle, medial femoral epicondyle and gastrocnemius tubercle. ${ }^{42}$ Since a non-anatomical reconstruction of the MPFL can lead to non-physiological patellofemoral loads and kinematics, the MPFL femoral insertion anatomy chosen was within the boundary of the medial epicondyle. ${ }^{43}$ The MPFL was initially tensioned such that the tension was minimal across passive flexion from $5^{\circ}$ to $70^{\circ}$. The initial tension set at $5^{\circ}$ flexion was set to $8 \mathrm{~N}$, this ensured the MPFL was engaged at and near full extension, ${ }^{38}$ but also allowed for a slack MPFL later in flexion when the patella engages the trochlear groove, minimizing unwanted PF contact stress. ${ }^{32}$

Radial basis functions (RBF) have been used to morph whole femur geometry; ${ }^{44}$ here this approach was used to perform sulcus deepening trochleoplasty. Landmarks were chosen as the three points used in calculating the sulcus angle. The deepest point in the trochlear groove was moved inward to create a sulcus angle of $138^{\circ}$, the average angle reported in a control population. ${ }^{45}$ Trochlear deepening was simulated with RBF points selected with the knee flexed 
This is an author-produced, peer-reviewed version of this article. The final, definitive version of this document can be found online at Journal of Orthopaedic Research, published by Wiley Periodicals, Inc. on behalf of the Orthopaedic Research Society. Copyright restrictions may apply. https://doi.org/10.1002/jor.24526

at $0^{\circ}, 30^{\circ}, 60^{\circ}$ and $90^{\circ}$ to allow for smooth modification of the patient-specific femur bone and cartilage along the entire trochlear groove. TT-D surgery was virtually performed by superior-inferior (S-I) translation of the tibial tubercle ${ }^{39}$ which is the insertion site of the patellar tendon on the tibia. The tibial tubercle, and thus the patella and attached ligament and tendons, were translated distally. The tubercle was moved distally to create an Insall-Salvati ratio with the original tibial tubercle attachment site of 1.07 , the average value for a healthy control. ${ }^{19}$ TT-M surgery was virtually performed through medial transfer of the tibial tubercle, for a post-operative TT-TG distance of 12 mm. ${ }^{19,21,39}$

\section{$\underline{\text { Statistical Analysis }}$}

The force-mobility results between the surgeries performed at varying prescribed flexion angles were analyzed using SPSS (version 25; SPSS Inc, Chicago, Illinois) using two-way repeated measures ANOVA. The threshold for statistical significance was set at $p=0.05$. A post hoc test was performed to compare the outcomes of the different surgeries, with a Bonferroni correction applied to control the familywise error rate. Linear regression was used to study the linear relationship between flexion and MPFL load, and flexion and trochlear constraint force.

\section{Results}

The sulcus angle before surgery had a mean and standard deviation of $167.7^{\circ} \pm 5.4^{\circ}$ [range: $158^{\circ}, 177^{\circ}$ ]. The InsallSalvati ratio before surgery was $1.28 \pm 0.20[0.83,1.85]$. The TT-TG distance before surgery was $19 \pm 4[8,26] \mathrm{mm}$. Each subject-specific model underwent multiple surgical combinations of MPFL reconstruction, tibial tubercle osteotomy, and trochleoplasty. Classification of knees ranged from eight knees with three abnormal factors to two knees with no abnormal factors (Table 1).

Differences in patellar I-E rotation and patellar M-L alignment were largest between the preoperative group and the equivalent healthy controls, with increasing differences later in flexion (Figure 5). 25 patient-specific models (89\%) experienced patellar dislocation in preoperative simulations, particularly in later $\left(30^{\circ}\right.$ and $\left.40^{\circ}\right)$ flexion, compared to $0 \%$ across postoperative simulations. Comparing between surgeries, restorative MPFLR to pre-injury state resulted in the most patellar I-E rotation, M-L translation and MPFL reaction force across all flexion angles, while the equivalent healthy control group had the least patellar I-E and M-L motion (Figure 5). Across all surgeries, with increasing flexion, the MPFL reaction load decreased $(\mathrm{r}=-0.56, p<0.01)$ while, in general, the trochlear constraint force increased $(\mathrm{r}=0.45, p<0.01)$ (Figures 5 and 6$)$.

There was a significant main effect of surgery on patellar I-E rotation $(p<0.001)$, patellar M-L alignment $(p<0.001)$, MPFL load $(p<0.001)$, and trochlear constraint force $(p=0.016)$. Of particular interest, the Bonferroni post-hoc test revealed patellar I-E rotation and patellar M-L alignment for the preoperative group was much larger for all flexion angles and significantly different when compared to all surgeries $(p<0.007)$. When comparing the healthy equivalent control group, there were no significant differences across all metrics when compared to the patient-specific reconstruction ( $p>0.05)$. The equivalent healthy control group was significantly different from the generic (MPFLR and MPFLR+TT-M) and less invasive patient-specific surgery groups when comparing patellar I-E rotation and MPFL load. Furthermore, the less invasive patient-specific surgery was not significantly different when compared to tibial tubercle osteotomy (MPFLR+TT-M). There were no significant differences in trochlear constraint force except between less invasive surgery and the pre-operative group $(\mathrm{p}=0.017)$.

There was a significant main effect of flexion angle on patellar I-E rotation $(p=0.03)$, MPFL load $(p<0.001)$, trochlear constraint force $(p<0.001)$, but not on patellar M-L alignment $(p=0.36)$. The Bonferroni post-hoc test showed that MPFL load between $30^{\circ}$ and $40^{\circ}(\mathrm{p}=0.17)$ were not significantly different, while all other combinations of flexion angle were significantly different $(\mathrm{p}<0.05)$ with increasing MPFL load as TF flexion angle decreased. The Bonferroni correction factor in the post-hoc test led to no detection in significant differences in patellar I-E rotation when comparing each combination of flexion angle. The trochlear constraint force between $20^{\circ}$ and $30^{\circ}(\mathrm{p}=0.30)$, and $30^{\circ}$ and $40^{\circ}(\mathrm{p}=0.47)$ were not significantly different, while all other combinations of flexion angle were significantly different $(\mathrm{p}<0.05)$ with increasing trochlear constraint force as TF flexion angle increased. 
This is an author-produced, peer-reviewed version of this article. The final, definitive version of this document can be found online at Journal of Orthopaedic Research, published by Wiley Periodicals, Inc. on behalf of the Orthopaedic Research Society. Copyright restrictions may apply. https://doi.org/10.1002/jor.24526

There was a significant interaction effect between flexion angle and surgery on patellar I-E rotation $(p<0.001)$ and patellar M-L alignment $(p<0.001)$, but not on MPFL load $(p=0.77)$ and trochlear constraint force $(p=1.00)$. The significant interaction effect indicates the different groups responded differently by flexion angle. In particular, the preoperative group had on average larger patellar I-E rotation and patellar M-L alignment later in flexion, while each surgery group was consistent across all flexion angles.

\section{Discussion}

The preoperative group had the largest amount of patellar I-E rotation and patellar M-L alignment, which are strong indicators of PF instability. 25 of the 28 patients in the preoperative group experienced lateral patellar dislocation in later flexion. The healthy equivalent control group, which restored all anatomic values to the average of a healthy control population, consistently resulted in the least amount of patellar I-E rotation, M-L translation, and MPFL restraining load during lateral displacement tests. MPFLR alone to restore pre-injury state performed the poorest of all surgeries on these three stability metrics; however, it was significantly better than preoperative simulations. For all simulation groups, MPFL reaction load decreased with increasing flexion, while, in general, trochlear constraint force increased, indicating the patella is being restrained by soft tissue early in flexion, and by bony anatomic structures later in flexion, which is consistent with the literature ${ }^{38}$ The less invasive patient-specific surgery was not significantly different when compared to a generic tibial tubercle osteotomy, which indicates neglecting the most invasive procedure in a surgery is no more effective in stabilizing the knee as a generic approach. Patient-specific reconstruction was the only surgical group with results statistically comparable to the healthy equivalent control group across all metrics. This indicates a targeted subject-specific approach to correct for abnormal factors may be necessary to best restore normal PF stability in patients suffering from recurrent patellar dislocation.

To date, there is little consensus in the clinical outcomes of different types of treatment and hence best practice in the treatment of patellar dislocation remains uncertain. Few studies have compared outcomes of both soft tissue and bony structure surgeries. In previous computational studies, Elias et al. assessed knee kinematics and mechanics for various MPFLR graft tensions ${ }^{14}$ and the relationship between anatomy and dynamic patellar tracking for different MPFLR conditions. ${ }^{13}$ Others simulated knee function of different MPFL reconstruction femoral insertion conditions, ${ }^{12,46}$ which found as little as $5 \mathrm{~mm}$ malposition of MPFL insertion largely increased MPFL tension. In our study, each patient had the same MPFL insertion for each surgery to eliminate this source of variability. In this study, tibial tubercle osteotomy and trochlear groove deepening were reconstructed to that of a control population on a per patient basis to improve patient patellar stability. In previous in-vitro studies, researchers assessed the stability of the knee by loading the quadriceps and applying a lateral load to the patella. Amis et al. studied the effect of trochleoplasty in initially healthy normal cadaveric knees which were artificially made dysplastic. ${ }^{1}$ Senavongse et al. studied vastus medialis obliquus malfunction, sulcus angle, and the medial retinacular structure. ${ }^{17}$ Ostermeier et al. compared the effects of two different techniques of medial patellofemoral ligament (MPFL) reconstruction and proximal soft tissue realignment. ${ }^{16}$ Our study focused on common soft tissue and bony structure patellar stabilization procedures, including MPFLR, trochleoplasty, and tibial tubercle osteotomy in a computational environment which allowed for direct quantitative comparison between different potential interventions. This data may guide clinical decisions in the type of surgical reconstruction that is required to restore patient anatomy to levels which produce patellar motion and restraint comparable to those of control subjects.

There are a number of limitations and simplifications associated with this study. Trochleoplasty was simulated by deepening the trochlear groove. In clinical practice, in addition to altering the anterior-posterior depth of the groove, it may also be set more laterally, simultaneously altering the TT-TG distance. ${ }^{21}$ However, in this study we isolated each variable, such that each surgery addresses a single abnormal factor. A similar computational approached may be employed in future work to evaluate these combined changes on patellar stability. This is a purely computational analysis of an isolated PF model with prescribed TF alignment applied across analyses. Prior work had been performed to demonstrate validity of this model for a healthy knee. The PF model has shown fidelity in reproducing patellar kinematics, with average root mean square (RMS) differences of less than $3.1^{\circ}$ and $1.7 \mathrm{~mm}$ for rotations and translations, respectively. ${ }^{47,48}$ However, TF kinematics are likely quite variable across this patient population, and this would alter the orientation of the patellar tendon, affecting PF kinematics and MPFL load. Unfortunately, patientspecific TF kinematics were not available for this patient group. It is possible that with significant external femoral IE rotation, tibial tubercle osteotomy and MPFLR may become more impactful on restoring patellar stability, however, for smaller variation in TF kinematics we anticipate there would be little change in the surgical trends reported here. 
This is an author-produced, peer-reviewed version of this article. The final, definitive version of this document can be found online at Journal of Orthopaedic Research, published by Wiley Periodicals, Inc. on behalf of the Orthopaedic Research Society. Copyright restrictions may apply. https://doi.org/10.1002/jor.24526

The protocol of using a consistent TF kinematic condition is in alignment with assessing stability in cadaver studies. ${ }^{1,16,15}$ In future work the computational framework described here may be used to conduct a sensitivity study to comprehensively quantify the role of TF kinematics on patellar stability.

The quantitative nature of this computational study allows for reasonable comparative analysis between different surgical intervention options. However, there are likely loading conditions that would further predispose a subject to patellar instability. This study did not account for articular cartilage injury that can occur with dislocation and adversely affect stability. The $50 \mathrm{~N}$ lateral load applied to the patella did not dislocate any of the patients who underwent surgery, however it still gave valuable information on patellar tracking. Given similar but different loading, we can expect similar trends in outcomes. There were no corrective surgeries without MPFLR, but after review of the literature, ${ }^{10}$ MPFLR is recommended with or without other restorative procedures. The surgeries performed in this study is likely not exhaustive, however it does address the three anatomic factors that are commonly cited in the literature.

The MPFLR simulated in this study represented reconstruction to the native MPFL state, with force-length characteristics calibrated to native MPFL properties and attachment sites ${ }^{41,43}$ This serves to represent a procedure that restores the knee to its intact, pre-injury state. The MPFLR simulated here is more similar to a MPFL repair procedure that a MPFL graft. In clinical practice, a MPFL graft has stiffer behavior than the native condition simulated in this study which may affect the results presented in this work. Additionally, attachment location may vary from the native position. In unpublished work, we evaluated the impact of these modeling decisions on patellar stability metrics using a single representative patient with patellar stability metrics that were closest to average across the cohort. MPFL stiffness was perturbed in 5\% increments up to $20 \%$. Femoral attachment sites was perturbed in $1.5 \mathrm{~mm}$ increments up to $6 \mathrm{~mm}$. For every 5\% increase in MFPL stiffness there was a corresponding change in final I-E and M-L position, MPFL force, and trochlear constraint force of $0.14^{\circ}, 0.07 \mathrm{~mm}, 1.6 \mathrm{~N}$ and $0.8 \mathrm{~N}$, respectively. For every $1.5 \mathrm{~mm}$ posterior change in femoral attachment there was a corresponding change in final I-E and M-L position, MPFL force, and trochlear constraint force of $0.84^{\circ}, 0.46 \mathrm{~mm}, 7.8 \mathrm{~N}$ and $15.4 \mathrm{~N}$. While these decisions do impact the magnitude of patellar stability metrics, each patient model was simulated with the same MPFL behavior across all virtual surgeries, and so we expect that the trends shown here are consistent if different MPFL behavior were simulated.

Many surgeons are reluctant to recommend surgery after primary dislocation. ${ }^{5,6}$ Some of these corrective surgeries, specifically, trochleoplasty, are technically challenging, and this technical complexity has not been accounted for in the current study. However, surgical simulation is safe, cost-effective and personalized. This computational approach can provide the surgeon with quantitative information on the potential benefit of a particular corrective surgery, so that they may make an informed decision as to whether the technical challenge is worth the improvement in stability. However, further in vivo work is needed to validate the surgical simulation on actual pre and post-operative clinical outcomes.

The results of this study suggest reconstructive surgery which corrects underlying anatomic abnormalities significantly improves joint stability during lateral displacement tests when compared to restorative soft-tissue or generic procedures. This foundational work may provide guidance to clinicians in treatment of patients with recurrent patellar dislocation. 
This is an author-produced, peer-reviewed version of this article. The final, definitive version of this document can be found online at Journal of Orthopaedic Research, published by Wiley Periodicals, Inc. on behalf of the Orthopaedic Research Society. Copyright restrictions may apply. https://doi.org/10.1002/jor.24526

\section{References}

1 Amis AA, Oguz C, Bull AM, et al. 2008. The effect of trochleoplasty on patellar stability and kinematics: a biomechanical study in vitro. J Bone Joint Surg Br 90: 864-869.

2 Andrish J. 2008. The management of recurrent patellar dislocation. Orthop Clin North Am 39: 313-327.

3 Gross RM. 1986. Acute dislocation of the patella: the Mudville mystery. Report of five cases. J Bone Joint Surg Am 68: 780-781.

4 Sanders TL, Pareek A, Johnson NR, et al. 2017. Patellofemoral Arthritis After Lateral Patellar Dislocation: A Matched Population-Based Analysis. Am J Sports Med 45: 1012-1017.

5 Atkin DM, Fithian DC, Marangi KS, et al. 2000. Characteristics of patients with primary acute lateral patellar dislocation and their recovery within the first 6 months of injury. Am J Sports Med 28: 472-479.

6 Hing CB, Smith TO, Donell S, Song F. 2011. Surgical versus non-surgical interventions for treating patellar dislocation. Cochrane Database Syst Rev: Cd008106.

7 Desio SM, Burks RT, Bachus KN. 1998. Soft tissue restraints to lateral patellar translation in the human knee. Am J Sports Med 26: 59-65.

8 Dopirak R, Adamany D, Bickel B, Steensen R. 2008. Reconstruction of the medial patellofemoral ligament using a quadriceps tendon graft: a case series. Orthopedics 31: 217.

9 Conlan T, Garth WP, Jr., Lemons JE. 1993. Evaluation of the medial soft-tissue restraints of the extensor mechanism of the knee. J Bone Joint Surg Am 75: 682-693.

10 Feller JA. 2015. Recurrent Patellar Instability: Assessment and DecisionMaking. Operative Techniques in Sports Medicine 23: 68-76.

11 Elias JJ, Jones KC, Copa AJ, Cosgarea AJ. 2018. Computational simulation of medial versus anteromedial tibial tuberosity transfer for patellar instability. J Orthop Res 36: 3231-3238.

12 DeVries Watson NA, Duchman KR, Bollier MJ, Grosland NM. 2015. A Finite Element Analysis of Medial Patellofemoral Ligament Reconstruction. Iowa Orthop J 35: 13-19.

13 Elias JJ, Jones KC, Cyrus Rezvanifar S, et al. 2018. Dynamic tracking influenced by anatomy following medial patellofemoral ligament reconstruction: Computational simulation. Knee 25: 262-270.

Elias JJ, Jones KC, Lalonde MK, et al. 2018. Allowing one quadrant of patellar lateral translation during medial patellofemoral ligament reconstruction successfully limits maltracking without overconstraining the patella. Knee Surg Sports Traumatol Arthrosc 26: 2883-2890.

Christoforakis J, Bull AM, Strachan RK, et al. 2006. Effects of lateral retinacular release on the lateral stability of the patella. Knee Surg Sports Traumatol Arthrosc 14: 273-277. Ostermeier S, Holst M, Bohnsack M, et al. 2007. In vitro measurement of patellar kinematics following reconstruction of the medial patellofemoral ligament. Knee Surg Sports Traumatol Arthrosc 15: 276-285. Senavongse W, Farahmand F, Jones J, et al. 2003. Quantitative measurement of patellofemoral joint stability: force-displacement behavior of the human patella in vitro. J Orthop Res 21: 780-786. Balcarek P, Zimmermann F. 2019. Deepening trochleoplasty and medial patellofemoral ligament reconstruction normalize patellotrochlear congruence in severe trochlear dysplasia. Bone Joint J 101-b: 325-330.

19 Steensen RN, Bentley JC, Trinh TQ, et al. 2015. The prevalence and combined prevalences of anatomic factors associated with recurrent patellar dislocation: a magnetic resonance imaging study. Am J Sports Med 43: 921-927.

Dejour H, Walch G, Nove-Josserand L, Guier C. 1994. Factors of patellar instability: an anatomic radiographic study. Knee Surg Sports Traumatol Arthrosc 2: 19-26.

21 Dejour D, Byn P, Ntagiopoulos PG. 2013. The Lyon's sulcus-deepening trochleoplasty in previous unsuccessful patellofemoral surgery. Int Orthop 37: 433-439.

22 Reddy KR, Reddy NS. 2012. Trochleoplasty and medial patellofemoral ligament reconstruction for recurrent patellar dislocation. Indian J Orthop 46: 242-245.

23 Zimmerer A, Sobau C, Balcarek P. 2018. Recent developments in evaluation and treatment of lateral patellar instability. J Exp Orthop 5: 3. Dislocations in Skeletally Mature Patients. Am J Orthop (Belle Mead NJ) 46: E86-e96.

Balcarek P, Rehn S, Howells NR, et al. 2017. Results of medial patellofemoral ligament reconstruction compared with trochleoplasty plus individual extensor apparatus balancing in patellar instability caused by severe trochlear dysplasia: a systematic review and meta-analysis. Knee Surg Sports Traumatol Arthrosc 25: 3869-3877. 
This is an author-produced, peer-reviewed version of this article. The final, definitive version of this document can be found online at Journal of Orthopaedic Research, published by Wiley Periodicals, Inc. on behalf of the Orthopaedic Research Society. Copyright restrictions may apply. https://doi.org/10.1002/jor.24526

26 Cohen ZA, Henry JH, McCarthy DM, et al. 2003. Computer simulations of patellofemoral joint surgery. Patient-specific models for tuberosity transfer. Am J Sports Med 31: 87-98.

27 Nelitz M, Dreyhaupt J, Lippacher S. 2013. Combined trochleoplasty and medial patellofemoral ligament reconstruction for recurrent patellar dislocations in severe trochlear dysplasia: a minimum 2-year follow-up study. Am J Sports Med 41: 1005-1012.

28 Steensen RN, Dopirak RM, Maurus PB. 2005. Minimally invasive "crescentic" imbrication of the medial patellofemoral ligament for chronic patellar subluxation. Arthroscopy 21: 371-375.

29 Arendt EA, Donell ST, Sillanpää PJ, Feller JA. 2017. The management of lateral patellar dislocation: state of the art. 2: 205-212.

30 Longo UG, Berton A, Salvatore G, et al. 2016. Medial Patellofemoral Ligament Reconstruction Combined With Bony Procedures for Patellar Instability: Current Indications, Outcomes, and Complications. Arthroscopy 32: 1421-1427.

31 Weber AE, Nathani A, Dines JS, et al. 2016. An Algorithmic Approach to the Management of Recurrent Lateral Patellar Dislocation. J Bone Joint Surg Am 98: 417-427.

32 Ronga M, Oliva F, Longo UG, et al. 2009. Isolated medial patellofemoral ligament reconstruction for recurrent patellar dislocation. Am J Sports Med 37: 1735-1742.

33 Song JG, Kang SB, Oh SH, et al. 2016. Medial Soft-Tissue Realignment Versus Medial Patellofemoral Ligament Reconstruction for Recurrent Patellar Dislocation: Systematic Review. Arthroscopy 32: 507-516.

34 Feller JA, Richmond AK, Wasiak J. 2014. Medial patellofemoral ligament reconstruction as an isolated or combined procedure for recurrent patellar instability. Knee Surg Sports Traumatol Arthrosc 22: 2470-2476. Fitzpatrick CK, Steensen RN, Tumuluri A, et al. 2016. Computational analysis of factors contributing to patellar dislocation. J Orthop Res 34: 444-453.

36 Elias JJ, Cosgarea AJ. 2007. Computational modeling: an alternative approach for investigating patellofemoral mechanics. Sports Med Arthrosc Rev 15: 89-94.

37 Arendt EA, England K, Agel J, Tompkins MA. 2017. An analysis of knee anatomic imaging factors associated with primary lateral patellar dislocations. Knee Surg Sports Traumatol Arthrosc 25: 3099-3107. Farahmand F, Naghi Tahmasbi M, Amis A. 2004. The contribution of the medial retinaculum and quadriceps muscles to patellar lateral stability--an in-vitro study. Knee 11: 89-94.

39 Duthon VB. 2015. Acute traumatic patellar dislocation. Orthop Traumatol Surg Res 101: S59-67.

40 Sallay PI, Poggi J, Speer KP, Garrett WE. 1996. Acute dislocation of the patella. A correlative pathoanatomic study. Am J Sports Med 24: 52-60.

41 Atkinson P, Atkinson T, Huang C, Doane R. in Orthopaedic Research Society 2000 (Orlando, Florida, 2000).

42 Aframian A, Smith TO, Tennent TD, et al. 2017. Origin and insertion of the medial patellofemoral ligament: a systematic review of anatomy. Knee Surg Sports Traumatol Arthrosc 25: 3755-3772. Schottle P, Schmeling A, Romero J, Weiler A. 2009. Anatomical reconstruction of the medial patellofemoral ligament using a free gracilis autograft. Arch Orthop Trauma Surg 129: 305-309.

44 Grassi L, Hraiech N, Schileo E, et al. 2011. Evaluation of the generality and accuracy of a new mesh morphing procedure for the human femur. Med Eng Phys 33: 112-120.

45 Shen J, Qin L, Yao WW, Li M. 2017. The significance of magnetic resonance imaging in severe femoral trochlear dysplasia assessment. Exp Ther Med 14: 5438-5444.

46 Elias JJ, Cosgarea AJ. 2006. Technical errors during medial patellofemoral ligament reconstruction could overload medial patellofemoral cartilage: a computational analysis. Am J Sports Med 34: 1478-1485.

47 Baldwin MA, Clary C, Maletsky LP, Rullkoetter PJ. 2009. Verification of predicted specimen-specific natural and implanted patellofemoral kinematics during simulated deep knee bend. J Biomech 42: 23412348.

48 Besier TF, Draper CE, Gold GE, et al. 2005. Patellofemoral joint contact area increases with knee flexion and weight-bearing. J Orthop Res 23: 345-350.

\section{List of Tables:}

Table 1: Prevalence and combined prevalence of abnormal anatomic factors. The following abbreviations were used to identify subjects with abnormal factors: $\mathrm{P}$ - patella alta (Insall-Salvati ratio $\geq 1.2$ ); $\mathrm{Q}$ - abnormally increased TTTG distance (TT-TG distance $\geq 20 \mathrm{~mm}$ ); $\mathrm{S}$ - trochlear dysplasia (flat or convex trochlear groove). 
This is an author-produced, peer-reviewed version of this article. The final, definitive version of this document can be found online at Journal of Orthopaedic Research, published by Wiley Periodicals, Inc. on behalf of the Orthopaedic Research Society. Copyright restrictions may apply. https://doi.org/10.1002/jor.24526

\section{List of Figures:}

Figure 1: Finite element model including femur, tibia, patella bone, and quadriceps muscle representations (RF, VI, VL and VM) with centroid-path lines of action (left). Detail patellofemoral joint with cartilage and patellar and quadriceps tendons (center). Femoral superior-inferior, medial-lateral, and anterior-posterior axes (center, right).

Figure 2: Metrics used to assess the stability of the knee at various flexion angles after a $50 \mathrm{~N}$ lateral load is applied to the patella.

Figure 3: Measurement of anatomic factors: sulcus angle (left), Insall-Salvati ratio (center), and tibial tubercletrochlear groove distance (right).

Figure 4: Patient-specific 3D dynamic FE models were developed to perform virtual surgeries including MPFL reconstruction, tibial tubercle medial / distal transfer (TT-M / TT-D), and trochleoplasty (TP).

Figure 5: Top: Comparison of indicators of patellar instability in pre-operative, restorative surgery, generic TT osteotomy, patient-specific reconstruction, patient-specific less invasive surgery and equivalent health control with \pm 0.5 standard deviation (STD) shaded regions. Bottom: PF pose after a $50 \mathrm{~N}$ lateral displacement is applied to the patella for preoperative condition (grey), restorative surgery (blue) and equivalent healthy control (red).

Figure 6: Patellar I-E rotation and M-L alignment for each post-operative group with \pm 0.5 standard deviation (STD) shaded regions.

Table 1: Prevalence and combined prevalence of abnormal anatomic factors. The following abbreviations were used to identify subjects with abnormal factors: $\mathrm{P}$ - patella alta (Insall-Salvati ratio $\geq 1.2$ ); $\mathrm{Q}$ - abnormally increased TTTG distance (TT-TG distance $\geq 20 \mathrm{~mm}$ ); $\mathrm{S}$ - trochlear dysplasia (flat or convex trochlear groove).

\begin{tabular}{|l|l|l|l|}
\hline Factors & Number in group (\%) & Patient-specific surgery & Less invasive surgery \\
\hline PQS & $8(28.6)$ & MPFLR, TT-M,TT-D+TP & MPFLR, TT-M+TT-D \\
\hline PS & $5(17.9)$ & MPFLR,TT-D+TP & MPFLR+TT-D \\
\hline S & $5(17.9)$ & MPFLR+TP & MPFLR \\
\hline QS & $3(10.7)$ & MPFLR, TT-M+TP & MPFLR+TT-M \\
\hline PQ & $3(10.7)$ & MPFLR, TT-M+TT-D & MPFLR \\
\hline P & $2(7.1)$ & MPFLR+TT-D & MPFLR \\
\hline No factors & $2(7.1)$ & MPFLR & Not Applicable \\
\hline
\end{tabular}

